STRUCTURAL SCIENCE CRYSTAL ENGINEERING MATERIALS

ISSN 2052-5206

Received 20 November 2015

Accepted 24 November 2015

Keywords: dynamical diffraction; electron diffraction tomography; electron crystallography.

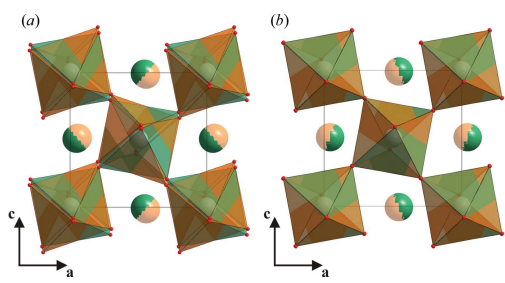

C 2015 International Union of Crystallography

\section{Closing the gap between electron and X-ray crystallography}

\author{
Enrico Mugnaioli*
}

Dipartimento di Scienze Fisiche, della Terra e dell'Ambiente, Università degli Studi di Siena, Via Laterino 8, 53100 Siena, Italy. *Correspondence e-mail: enrico.mugnaioli@unisi.it

The year 2014 celebrated the centenary of von Laue's discovery of X-ray scattering by crystals, which opened the way for understanding how matter is organized at the atomic level. The fact that accelerated electrons are also scattered by crystalline matter in a similar way was discovered just 13 years later (Davisson \& Germer, 1927), and by the 1930s the first attempts at using electron diffraction for structure determination were reported (e.g. Rigamonti, 1936).

Accelerated electrons have three main differences with respect to X-rays. Their wavelength is 1-2 orders of magnitude shorter, in principle allowing for better resolution and normally triggering the excitation of several reflections at the same time. Accelerated electrons also have a higher cross-section with matter, assuring a good signal-to-noise information for nanoscopic volumes. However, the short wavelength and the strong interaction are also weaknesses, because they favor multiple scattering events (usually referred to as dynamical effects), which violate the kinematical assumption that $\left|F_{h k l}\right|^{2}$ is directly proportional to observed $I_{h k l}$. Finally, electrons are charged and it is relatively easy to deflect them by electromagnetic coils. The ability to focus the beam into a nanometric probe allows structural (and chemical) information to be obtained for areas of a few squared nanometers.

The main advantage of using accelerated electrons as scattered radiation is the evident possibility of collecting three-dimensional single-crystal diffraction data from submicroscopic grains. Crystals of such dimensions are untreatable by single-crystal X-ray techniques, while X-ray powder diffraction (XRPD) has the obvious limitations that the signal comes from multiple crystallites and that it is projected in one dimension. These restrictions are critical when the sample is polyphasic or when the structure of interest is characterized by large cell parameters or pseudo-symmetry, causing accidental and systematic overlap of symmetrically independent reflections at medium-high resolution. In fact, XRPD cannot be used for structure determination or refinement of minor components in polyphasic samples, components that are often critical for establishing the chemical, physical and clinical properties of the entire material.

Despite the appealing potentialities, for a long time electron crystallography was almost neglected due to the fact that even with samples thinner than $100 \mathrm{~nm}$ dynamical effects introduce significant perturbations on reflection intensities, in certain cases making it difficult even to distinguish between weak and strong reflections. Early on, two theoretical approaches - multislice numerical calculations (Cowley \& Moodie, 1957) and Bloch wave analytical treatment (Bethe, 1928) - were developed to describe electron multiple scattering. It has been shown that by combining dynamical formalism with convergent-beam electron diffraction (CBED), structure parameters can be refined with high accuracy (Tsuda \& Tanaka, 1999), and eventually it is possible to establish the orientation of electronic $d$-orbitals of transition metal atoms in simple oxide structures (Zou et al., 1999; Tsuda et al., 2010). Nevertheless, up to now this methodology did not find a common use for the investigation of more complex unknown compounds.

Starting from the 1980s, several ab initio structure determinations were obtained on the basis of conventional in-zone electron diffraction patterns, adopting the simple kinematical assumption (e.g. Weirich et al., 1996). However, this approach proved successful only in a limited number of cases, and in any case required a long time and high expertise for a satisfactory data collection and the interpretation of the attained solution (Dorset et al., 2005). 
A pivotal impulse for electron crystallography was provided by the development of tomographic data collection routines (Kolb et al., 2007). Electron diffraction tomography (EDT) involves sampling of the whole available reciprocal space in steady steps, neglecting the orientation of low-index diffraction zones (Kolb et al., 2011). Highly complete and quasikinematical data sets can be produced by coupling EDT with precession of the beam (Vincent \& Midgley, 1994; Mugnaioli et al., 2009) or by operating a fine beam tilt scanning of the reciprocal space (rotation electron diffraction - RED; Zhang et al., 2010). Within a few years, EDT had permitted the $a b$ initio determination of several complex structures, both inorganic and organic, by employing a relatively straightforward and reproducible procedure (e.g. Jiang et al., 2011; Gorelik et al., 2012). Recently, Nannenga et al. (2014) and Gemmi et al. (2015) implemented a fast (continuous) EDT acquisition system, able to deliver electron diffraction data of sufficient quality for solving simple protein structures. All these achievements were remarkably obtained using the simple kinematical approximation, i.e. neglecting dynamical effects.

Nonetheless, the structure models thereby obtained are not equivalent to the ones refined against $\mathrm{X}$-ray diffraction data, whether in terms of precision, accuracy or self-validation. Internal and final structure residuals are typically in the range $20-40 \%$ for $R\left(F_{h k l}\right)$, while standard figures of merit cannot be used to evaluate the quality of the refinement. Differences in interatomic distances between expected structures and those solved and refined on the basis of electron diffraction data (using the kinematical approach) may be more than $0.5 \AA$ (Kolb et al., 2011), see Fig. 1(a). What is more, there is no parameter capable of ascertaining the quality or reliability of the model. Actually, models obtained ab initio are generally trustworthy for establishing the overall atomic connectivity, but cannot be kinematically refined in order to investigate fine structural features, like geometrical distortions, partial occupancies or thermal vibrations. Hence, the need of an external refinement and validation, usually obtained by Rietveld

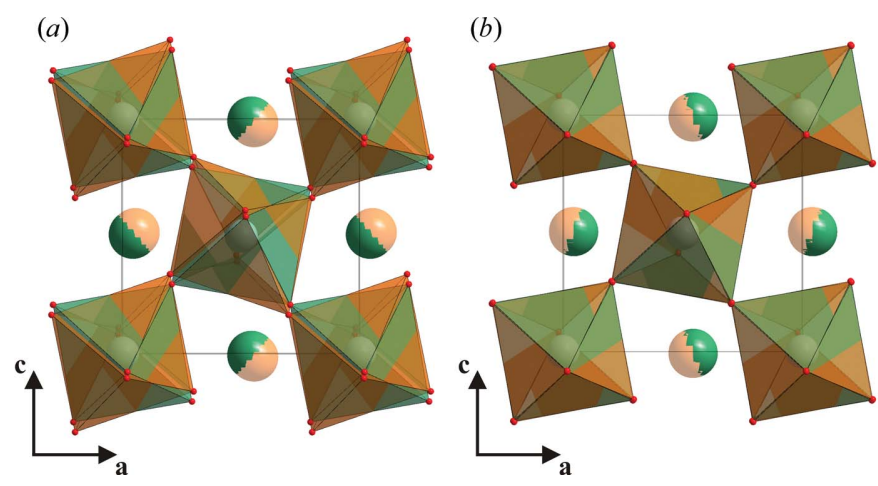

Figure 1

Comparison between the structure of $\mathrm{PrVO}_{3}$ refined against X-ray diffraction data (green) by Martínez-Lope et al. (2008) and against electron diffraction data (brown), using (a) the kinematical approach and (b) the dynamical formalism implemented by Palatinus et al. (2013). Pr atoms are represented by isolated spheres and $\mathrm{V}$ atoms inside $\mathrm{VO}_{6}$ octahedra. methods and XRPD data (still only for single-phase samples or main components of polyphasic mixtures).

The research of Lukas Palatinus and coworkers is directed towards the development of a method allowing structure refinement and self-validation by electron diffraction data alone. Other algorithms had been previously proposed with the same purpose (Jansen et al., 1998; Dudka et al., 2008; Oleynikov, 2011), but they did not find a widespread use, probably because they were limited to conventional in-zone diffraction patterns. Palatinus et al. (2013) demonstrated the feasibility of a robust structure refinement algorithm based on Bloch-wave theory and were able to account for dynamical effects (for brevity addressed as 'dynamical refinement'). The key development was the combination of the dynamical diffraction theory with the beam precession method (Vincent \& Midgley, 1994). Later, this dynamical refinement algorithm was further implemented in order to deal with off-zone EDT diffraction patterns (Palatinus, Petř́íček \& Corrêa, 2015). The paper published in the present issue (Palatinus, Corrêa et al., 2015) reports several cases of structures refined against precession EDT data. Results are indeed impressive, as structure residuals by electron diffraction achieve values hitherto exclusive to X-ray and neutron diffraction. At the same time, differences in interatomic distances between models refined against electron and X-ray or neutron diffraction data come in systematically below $0.06 \AA$ (Fig. $1 b$ ). Partial occupancies are also refined to reliable values. The effects of different experimental computing settings are also extensively investigated, confirming that the method is robust and able to deal with data sets of different completeness and resolution.

In recent years, EDT delivered for the first time a rather straight path for $a b$ initio structure determination from electron diffraction data. The availability of a proper refinement algorithm that takes into account the dynamical scattering and guarantees results approaching X-ray crystallography in terms of precision, accuracy and reliability now establishes a complete pathway for the structure characterization of single sub-micrometric and nanoscopic crystals. The availability of such a powerful 'structural nanoprobe' may open new horizons for the understanding of nanostructured materials and of processes operating at the nanoscale. This will likely create the opportunity for crucial advancements in several scientific disciplines, like natural sciences, materials science, pharmacy and bio-medicine.

\section{References}

Bethe, H. (1928). Ann. Phys. 392, 55-129.

Cowley, J. M. \& Moodie, A. F. (1957). Acta Cryst. 10, 609619.

Davisson, C. \& Germer, L. H. (1927). Phys. Rev. 30, 705740.

Dorset, D. L., Roth, W. J. \& Gilmore, C. J. (2005). Acta Cryst. A61, 516-527.

Dudka, A. P., Avilov, A. S. \& Lepeshov, G. G. (2008). Crystallogr. Rep. 53, 530-536. 


\section{scientific commentaries}

Gemmi, M., La Placa, M. G. I., Galanis, A. S., Rauch, E. F. \& Nicolopoulos, S. (2015). J. Appl. Cryst. 48, 718-727.

Gorelik, T. E., van de Streek, J., Kilbinger, A. F. M., Brunklaus, G. \& Kolb, U. (2012). Acta Cryst. B68, 171-181.

Jansen, J., Tang, D., Zandbergen, H. W. \& Schenk, H. (1998). Acta Cryst. A54, 91-101.

Jiang, J., Jorda, J. L., Yu, J., Baumes, L. A., Mugnaioli, E., DiazCabanas, M. J., Kolb, U. \& Corma, A. (2011). Science, 333, 11311134.

Kolb, U., Gorelik, T., Kübel, C., Otten, M. T. \& Hubert, D. (2007). Ultramicroscopy, 107, 507-513.

Kolb, U., Mugnaioli, E. \& Gorelik, T. E. (2011). Cryst. Res. Technol. 46, 542-554.

Martínez-Lope, M., Alonso, J., Retuerto, M. \& Fernández-Díaz, M. (2008). Inorg. Chem. 47, 2634-2640.

Mugnaioli, E., Gorelik, T. \& Kolb, U. (2009). Ultramicroscopy, 109, $758-765$.

Nannenga, B. L., Shi, D., Leslie, A. G. W. \& Gonen, T. (2014). Nat. Methods, 11, 927-930.

Oleynikov, P. (2011). Cryst. Res. Technol. 46, 569-579.
Palatinus, L., Corrêa, C. A., Steciuk, G., Jacob, D., Roussel, P., Boullay, P., Klementová, M., Gemmi, M., Kopeček, J., Domeneghetti, M. C., Cámara, F. \& Petříček, V. (2015). Acta Cryst. B71, 740-751.

Palatinus, L., Jacob, D., Cuvillier, P., Klementová, M., Sinkler, W. \& Marks, L. D. (2013). Acta Cryst. A69, 171-188.

Palatinus, L., Petříček, V. \& Corrêa, C. A. (2015). Acta Cryst. A71, 235-244.

Rigamonti, R. (1936). Gazz. Chim. Ital. 66, 174-182.

Tsuda, K., Morikawa, D., Watanabe, Y., Ohtani, S. \& Arima, T. (2010). Phys. Rev. B, 81, 180102.

Tsuda, K. \& Tanaka, M. (1999). Acta Cryst. A55, 939-954.

Vincent, R. \& Midgley, P. A. (1994). Ultramicroscopy, 53, 271282.

Weirich, T. E., Ramlau, R., Simon, A., Hovmöller, S. \& Zou, X. (1996). Nature, 382, 144-146.

Zhang, D., Oleynikov, P., Hovmöller, S. \& Zou, X. (2010). Z. Kristallogr. 225, 94-102.

Zou, J. M., Kim, M., O'Keefe, M. \& Spence, J. C. H. (1999). Nature, 401, 49-52. 